\title{
Use of Computer Software in Education of Students in Primary School and Preschool
}

\section{Dzhumaev Mamanazar Irgashevich* and Urinbaeva Lola Uktamovna}

Professor at the Tashkent State University named after Nizami. Tashkent city (Uzbekistan)

Senior lecturer at the Nizami Tashkent State University. Tashkent city (Uzbekistan)

*Corresponding author: Dzhumaev Mamanazar Irgashevich, professor at the Tashkent State University named after Nizami. Tashkent city (Uzbekistan)

\section{Annotation}

This work examines the Modern system of pre-school education, orienting society towards a new humanistic approach to the child as a developing individual, in need of understanding and respect for her interests and rights.

Keywords: intelligence; thinking; component; formation; concepts; thinking; intelligence; labor; learning

\section{Introduction}

The goal of the strategy for 2017-2021 is to fundamentally increase the effectiveness of the reforms, create conditions for the comprehensive and accelerated development of the state and society, implement priority directions for the modernization of the country and liberalize all spheres of life [1-4]. As noted in the document, a comprehensive analysis of the stage of independent development passed by Uzbekistan, as well as the changing world economy in the context of globalization, require the development and implementation of "radically new ideas and principles for the further sustainable and accelerated development of the country" [1].

The action strategy will be implemented in five stages, each of which provides for the approval of a separate annual State program for its implementation in accordance with the declared name of the year. The development of the social sphere, aimed at a consistent increase in employment and the implementation of targeted programs for the development of education, culture, science, literature, art and sports, and the improvement of state youth policy [3].

In the resolution of the Government of the Republic of Uzbekistan. The goal of the strategy, designed for 2017-2021, is a radical increase in the effectiveness of the reforms, creation of conditions for the comprehensive and accelerated development of the state and society, implementation of priority directions for the modernization of the country and liberalization of all spheres of life. As noted in the document, a comprehensive analysis of the stage of independent development passed by Uzbekistan, as well as the changing world economy in the context of globalization, require the development and implementation of "radically new ideas and principles for the further sustainable and accelerated development of the country"[1].

The action strategy will be implemented in five stages, each of which provides for the approval of a separate annual State program for its implementation in accordance with the declared name of the year. The development of the social sphere, aimed at a consistent increase in employment and the implementation of targeted programs for the development of education, culture, science, literature, art and sports, and the improvement of state youth policy; "Development of the social sphere of the Republic of Uzbekistan" $[5,6]$, the main directions of the development of education at school and approaches to learning are identified, which imply the orientation of instruction towards the formation of general intellectual, universal skills and methods of activity that have a practice-oriented orientation (competency-based approach to learning).

In determining the selection and location of the educational content of schoolchildren, the assessment of trends in the development of mathematical education is essential. Analysis of the traditional course of arithmetic and the course of mathematics of elementary school, conducted by A.M. Pyshkalo showed, "that they are built on two basic concepts of number and quantity, which are 
considered in the sequence" number quantity ". In the same scheme and course of mathematics in the experiment L.V. Zankova. Experimental course V.V. Davydov built according to the scheme "value ratio number." Moreover, the ratio refers to the number of measurements in the measured value. What causes researchers to question the sequence of study of concepts?

However, the measurement of a quantity during which a measure is postponed a certain number of times includes, as indicated by J. Piaget, two logical operations. The first is the separation process, which allows the child to understand that the whole consists of parts. The second is the replacement operation, which allows you to attach one part to another and in this way create a system of units. It is more difficult to divide a continuous whole into interchangeable units than to list them divided. Therefore, the dimension develops later than the concept of number.

Methodical classes, as the authors write, are, in fact, a system of didactic games, during which children explore problem situations, identify significant signs and relationships, make discoveries. Knowledge is not given in finished form, but through the process of independent discovery by a child of the features and properties of the studied objects and phenomena. A feature of the program is its focus on a deeper study of "objects and phenomena of the world: it prepares children for the perception and elementary understanding of the dialectical unity of the world in its quantitative and qualitative relationships." The authors understand that the necessary condition for successful learning is the creation of a personalityoriented approach to the preschool child, the creation of an atmosphere of goodwill in the educational process.

The concept of lifelong education (pre-school and primary school) notes that "variability brought to the preschool education an unjustified interest in subject-based learning", while "a balance of reproductive (reproducing the finished sample) and research activities, joint and independent forms of activity is required. As a result of the ability to follow the pattern, rule, and instruction that has developed in preschool age, the arbitrariness of mental processes and behavior is formed, and initiative in cognitive activity arises.

In any case, the program for the mathematical development of preschool children in the "School 2100 ..." system repeats at its core the content of the traditional methodology for the formation of elementary mathematical representations and organizes training on a visual and practical basis [5]. The tendency to master a larger number of supporting concepts in the selection of content does not seem to be random. After all, the more basic scientific concepts a student learns, the closer education is to what is called science. "It is possible that in the future the list of basic concepts will be modified or expanded," notes A.M. Breathing.

Features of the forms of children's thinking - visualeffective and visual-figurative - limit the possibility of successful teaching of mathematics in a fairly full form, however, the orientation of traditional teaching on the visual properties of objects is not at all necessary. Educators identify intellectual development with mental development or the development of mental structures. Psychologists believe that children discover the acquired knowledge and actions in cases that cause intellectual difficulties. The child cannot complete the task in ways known to him; he must find a new way to complete the task. Such tasks are called problematic, and situations that cause the need for thinking processes, problematic situations.

Intellectual activity corresponds to a high level of human development. It forms the basis of his theoretical activity, including the use of complex systems of symbolic formations, and involves a fairly high level of abstraction from objects of activity [4]. The scientific development of a preschool child should be based on a system of successive small intellectual tasks aimed at the formation of certain intellectual skills.

For example, teaching the concept of "multitude" can be carried out on small intellectual tasks that form the following skills:

1. select each element of the set;

2. indicate the essential feature of an individual element of the set;

3. highlight the essential feature of the set;

4. indicate the generalizing word, the characteristic property of the set;

5. compare the elements of the set by property (by color, shape, size ...);

6. select one item and many items;

7. we establish that the element belongs to the set;

8. indicate an extra element that does not belong to the set;

9. select a subset in the set;

10. we break the set into classes, groups of objects, etc.

Citation: Dzhumaev Mamanazar Irgashevich* and Urinbaeva .Use of Computer Software in Education of Students in Primary School and Preschool Op Acc J Bio Sci \& Res 7(1)-2021. 
The selection, sequence, completeness of the content of small intellectual tasks is a serious problem. In the process of research, intellectual tasks were identified in the sequence of studying support concepts: "a set of relationships on a set of correspondence, number of geometric figures, logic" [5].

Consistent and systematic training in intellectual tasks forms the skills and techniques of intellectual activity. In the established practice of teaching, general and special methods of intellectual activity "do not act as objects of special assimilation, their formation proceeds only in the course of assimilation of knowledge and with insufficient control by the educator.Such an organization of learning determines the corresponding course of the process of formation of the main types of mental activity: a great stretch in time, the presence of a variegated variety of intermediate stages, a large spread of the results achieved by individual students. In psychology, this poorly managed process of mastering concepts is actually identified with intellectual development" [7].

In the case of training in intellectual tasks, various types of intellectual activity become the subject of special assimilation. In this case, the main methods of mental activity are formed: recognition, comparison, identification, analysis, synthesis, generalization, analogy, classification, etc. Thus, the formation of intellectual skills provides the development of thought processes and the mental development of the personality as a whole.The formallogical side of the methods of mental activity is formed on the material of mathematical content. However, psychologists argue that, being formed on any one subject content, the mental action is further used as a ready-made method of thinking in the analysis of any area of reality. The identification of small intellectual (scientific mathematical) problems and the definition of intellectual (scientific) skills as special assimilation not only contributes to the mathematical development of the child, but also outlines a completely new picture of intellectual development as a whole [8-10].

So, in the basis of the mathematical development of a preschool child, mathematical knowledge proper can be put. Their selection should be made in accordance with the scientific content of "big mathematics". A sequence of small portions of educational material aimed at the formation of mathematical knowledge forms a sequence of intellectual (scientific) tasks. In each such task, certain types of intellectual activity become the subject of special assimilation. In accordance with the theory, not only the development of mathematical knowledge occurs, but also specific types of cognitive, mental activity and general educational methods of thinking are formed. This overcomes the gap between the orientation of the preschool child on specific objective methods of action and the installation for the elementary school student of action with abstract mathematical concepts. Children should be taught in small intellectual problems that are adequate to one or another mathematical essence, to one or another image, representation, concept [11-15].

As a procedural component of mathematical training, a methodological training system has been developed as a source of awareness of the mechanisms of formation of elementary mathematical representations in young children. The selected mathematical content of the student's mathematical preparation on the basis of constructing a graph of the logical structure of the three disciplines of the curriculum allows you to organize studies at the level of elementary portions of the educational material. As an objective measure of assessing the quality of knowledge, a test can serve. Obtaining the necessary coefficient of assimilation of knowledge is an objective result (goal) of assimilation of theoretical knowledge, which is specified when organizing control in the traditional way in exams and tests [16].

The real educational process of a pedagogical university considers lectures and classroom practical exercises as the main form of training. Traditional teaching methods are used to organize the educational process in effective didactic systems. A distinctive feature of such an organization is a stable and long-term feedback and directional information process. This is ensured by a system of developed teaching aids, including textbooks and teaching aids; mathematical dictations; notebooks with a printed base; cards of general and individual tasks; graphic and settlement-graphic works; control sections; tests, etc.

Designing an effective educational process using a complete system of developed teaching aids that ensure the teacher's work in lecture and practical classes in didactic monosystems ensures the assimilation of knowledge at the level of algorithmic activity. To bring the student to the level of creativity, it is necessary to use integrated technology. The pre-mathematical training carried out in kindergarten is part of the general preparation of children for school and consists in the formation of elementary mathematical representations in them. This process is associated with all aspects of the upbringing and educational work of a kindergarten and is primarily aimed at solving the problems of mental education and mathematical development of preschool children. Its distinctive features are the general 
developmental orientation, the connection with mental, speech development, gaming, domestic, labor [17-19].

When stating and realizing the tasks of pre-mathematical preparation of preschool children, take into account:

1. patterns of formation and development of cognitive activity, mental processes and abilities, the personality of the child as a whole;

2. age-related opportunities for preschoolers in the assimilation of knowledge and related skills;

3. The principle of continuity in the work of kindergarten and school.

In the process of pre-mathematical training, educational, educational and developmental tasks are solved in close unity and interconnection with each other. By acquiring mathematical ideas, the child gains the necessary sensory experience of orientation in the various properties of objects and the relationships between them, masters the methods and techniques of cognition, and applies the knowledge and skills formed during the training in practice. This creates the prerequisites for the emergence of a materialistic outlook, connects learning with the surrounding life, and fosters positive personality traits. Let us dwell on the main tasks of pre-mathematical training of children in kindergarten $[20,21]$.

1. Formation of a system of elementary mathematical representations in preschoolers. From the content side, the most important in the sense of the formation of primary simple representations are such fundamental mathematical concepts as "set", "relation", "number", "quantity". These concepts are widely represented in the initial training, but not in the direct sense, but from the point of view of the propaedeutics of formation, only an idea of them. Figuratively speaking, a child in kindergarten comprehends "sciences before science", and naturally this is due to the fact that, in their psychological structure, elementary mathematical representations have a figurative nature. The gradual complication of knowledge mastered by children consists in increasing both the volume of quantitative) spatial and temporal representations, and the degree and generalization. The system of knowledge and initial ideas about sets, relationships, numbers and quantities, although it is very limited, by the scope of learning opportunities for preschoolers, is significant for further mastering the concepts of school mathematics. Elementary mathematical representations are formed $\mathrm{n}$; the basis for children to learn in a certain sequence of methods of action (for example, it is proposed to lay out as many objects on a free strip as they are drawn on the sample, to put strips of different lengths on top of each other, pick up pictures with objects to the corresponding geometric figure, etc.) The methods of action are gradually becoming more complicated; by the end of training in the kindergarten, the simplest skills are developed for counting objects, measuring distances, volumes of liquids and bulk solids using conditional standards, and the ability to perform calculations when solving arithmetic problems in one action of addition and subtraction.

Elementary mathematical representations and the corresponding methods of action are the main components of the knowledge system for preschoolers.

The assimilation of various concepts related to the most complex branches of human knowledge should be based on sensory experience and worldly ideas, which are formed already in preschool age.

The main difference between a concept and a concept is, first of all, that the concept reflects the essential features of an object, abstracted from its other, non-essential properties. The representation reflects both essential and non-essential properties of the object in its direct perception.

In experimental studies (P. Ya. Halperin, L.F. Obukhova, etc.), the possibility of the formation of separate full-fledged mathematical concepts in preschoolers is shown, but this requires special conditions. Consider some conditions under which the assimilation of concepts and the development of conceptual thinking are possible.

A conceptual way of recognizing objects is possible based on the method of phased formation of mental actions (P. Ya. Halperin). This method is a certain sequence of actions: knowing the essential sign of the concept-104, the child identifies the properties of the subject in question and compares them with the essential sign of the concept, and then concludes whether the analyzed object belongs to this concept or not. First, the comparison of signs occurs mod the guidance of the teacher. Then the child himself, comparing the signs, reasoning out loud. At the next stage, comparing these signs, he reasones mentally, "to himself", according to the same scheme, which serves as the basis for speech. So, gradually, assimilating the sequence of actions reflected in the external and then internal speech, the child masters the way of bringing any object, property or phenomenon under study. A detailed judgment according to the pattern of actions taken gradually passes first into a plan of short speech "to oneself", and then into a plan of mental action. Now, having mastered the mode of action

Citation: Dzhumaev Mamanazar Irgashevich* and Urinbaeva .Use of Computer Software in Education of Students in Primary School and Preschool Op Acc J Bio Sci \& Res 7(1)-2021. 
and reasoning, the child will be able to solve any new problem on his own.

Learning, built on the method of phased development of mental actions, allows you to get closer to the formation of the concept of number, based on an understanding of the principle of maintaining volume, mass and quantity, to create the basis for the emergence of elements of theoretical thinking (L. F. Obukhova).

Increasing the level in the generalization of mathematical representations, the formation of mathematical concepts is promoted not only by the special organization of mental activity, but also by the use of special cognitive tools in the learning process: models, graphs, diagrams, etc. For example, a "ladder" made up of circles models cardinal and ordinal relations of natural numbers, four circles pink, white, blue and black - model parts of the day and so on.. formation of elementary mathematical concepts in preschool children $\mathrm{m}$ Jette be implemented in different ways. Since the experience and knowledge of children is small, training basically goes like this: first, with the help of an adult, specific knowledge is accumulated, and then they are generalized to the simplest rules and patterns. However, this necessary and important path for the mental development of young children also has its drawbacks: children cannot go beyond those isolated facts and cases on the basis of which they were summarized to generalizations; unable to analyze a wider range of knowledge, which limits the development of their independent thoughts and searches. Therefore, in teaching it is necessary to use another way, when thought and assimilation of knowledge go from general to particular. The learned rule, children must learn to apply in specific conditions.

A rational combination of these methods contributes to the highest mental and mathematical development of children. It is not always necessary to put a child in the position of a "discoverer", to lead him from single concrete knowledge to conclusions and generalizations. The child must learn to master and acquire the ready-made knowledge accumulated by mankind, value it, be able to use it to analyze both their experience and the facts and phenomena of life around them. For example, at a certain stage, preschoolers are introduced to quadrangles. Turning to children's experience, one can first propose to find and name those familiar figures that have four sides and four corners and can be assigned to quadrangles, and secondly, to find objects or parts of objects of a quadrangular shape (this concretization deepens knowledge children about this geometric figure).
Similarly, children are introduced to polygons. Concretizing their knowledge, preschoolers show and name triangles, squares, rectangles of different sizes, relating all these figures to polygons. The idea of a polygon, as it were, is built up over the whole variety of figures, limited by closed broken lines, right and wrong, large and small.

Therefore, for the development of children's mental abilities, it is necessary to use different ways, to bring them to understanding the unity of the general and the individual, the abstract and the concrete. Education in kindergarten is not only a communication of knowledge, but also the development of children's mental abilities, mechanisms of mental activity, which facilitates the transition from empirical knowledge to conceptual.

2. The formation of the prerequisites of mathematical thinking and individual logical structures necessary for mastering mathematics in school and general mental development. Mastering the initial mathematical concepts contributes to the improvement of the cognitive activity of the child as a whole and its individual sides, processes, operations, actions. The formation of the logical structures of thinking - classification, ordering, understanding the preservation of quantity, volume mass, etc., acts as an important independent feature of the general mental and mathematical development of a preschool child.

The process of forming elementary mathematical representations is built taking into account the level of development of a visual-effective and visual-figurative thinking of a preschooler and has as its goal the creation of prerequisites for the transition to more abstract forms of orientation in the environment. Mastering various practical ways of comparing, grouping objects by quantity, size, shape, spatial location actually lays the foundation for logical thinking. In the process of forming mathematical representations, preschoolers develop the ability to use indirect methods to evaluate various properties of objects (counting to determine quantity, measurement to determine quantities, etc.), anticipate the result, judge the initial data by the result, understand not only visible external relations and dependencies, but also some internal, the most significant. A definite result of the education of preschoolers is not only the formed system of mathematical representations, but also the foundations of visual-schematic thinking as a transitional stage from concrete to abstract. In children, the ability to analytic-synthetic and classifying activities, abstracting and generalizing is improving.

3. The formation of sensory processes and abilities. The main direction in teaching young children is the

Citation: Dzhumaev Mamanazar Irgashevich* and Urinbaeva .Use of Computer Software in Education of Students in Primary School and Preschool Op Acc J Bio Sci \& Res 7(1)-2021. 
implementation of a gradual transition from specific, empirical knowledge to a more general one. Empirical knowledge formed on the basis of sensory experience is a prerequisite and necessary condition for the mental and mathematical development of preschool children.

Already in early childhood, ideas about the environment, about the signs and properties of the objective world begin to take shape: the shape, size, spatial arrangement of objects and their quantity. The basis for young children to learn about the qualitative and quantitative signs of objects and phenomena is based on sensory processes: sensation, perception, and representation. The kid learns the properties and qualities of the subject in actions, in a practical way.

"The closet is behind you," they say to the child. "And where is it from behind: where is the back?" - the child specifies and presses his back to the closet in order to specifically feel, to know the spatial position of the object behind. "Find among the toys those that look like this triangle." The child, having carefully examined the triangle and examined it with his hands, quite easily searches for objects similar to a given shape.

Children are purposefully taught certain techniques and generalized methods of examination: tracing an object's contour with a hand and looking to identify the shape, "weighing" objects on the palms of both hands in order to compare their masses, applying or applying strips of paper to compare lengths, comparing elements of one group of objects with another to clarify the relationships "more", "less", "equal", etc. This is a comparison in form, size, quantity, comparison of the revealed signs with what is already in the child's experience. A higher level of orientation in quantitative, spatial and temporal relations is ensured by the ability to use generally accepted standards. The system of standards has developed in the socio-historical practice of man and represents ordered forms (geometric figures), values (measures of length, mass, volume, time, etc.) and other qualities. By mastering this kind of knowledge, the child gets a set of standards, or standards, with which he can compare any newly perceived quality, find him a place among others.

In preschool age, the development of sensory standards is carried out not only at the perceptual, but also at the intellectual level (L. A. Wenger). Young children master the individual elements of the system of standards, using the survey activities that adults taught them. Older preschoolers, using classification, come to the realization of the principle of constructing such systems. The work on the development and use by children of sensory standards in kindergarten is just beginning, a deeper familiarization with them takes place at school.

Sensory processes (perception, representation) and abilities (observation, eye) are also the basis of focused work carried out with children in line with their pre-mathematical training. A special organization of sensory experience creates the basis for indirect knowledge, prepares for the formation of mathematical concepts.

4. Expanding the vocabulary of children and improving coherent speech. The process of forming elementary mathematical representations involves the systematic assimilation and gradual expansion of the vocabulary, improving the grammatical structure and connectedness of speech.

With the help of words, a child reflects quantitative relations with many, one, not one, so many, how many, equally, more, less, etc., which are recognized as a result of direct actions when comparing individual objects and their aggregates. Borrowed from the speech of others, the numeral words are filled with meaning and are used for a specific purpose - to find out how many objects. When counting, the child learns on an intuitive level to coordinate the numeral with the noun in gender, number and case. Comparison of collections of objects by quantity, and later comparison of numbers requires the construction and use of rather complex speech structures. Not only the results of cognitive activity are clothed in a speech form, but also its methods. The child is required to tell what he did (for example, put 6 red circles on the top strip and 7 blue ones on the bottom) and what happened (there were more blue circles than red ones and red ones less than blue ones). The deeper the mathematical relationships, dependencies and relationships are realized, the more advanced tools are used to reflect them in speech.

Children are taught not only on the sensory level to recognize the values of objects, but also to correctly reflect their ideas in a word, for example: wider - narrower, higher - lower, thicker - thinner, etc., distinguishing these changes from changes in the total volume (more - less, big small). Such differentiation is quite accessible to children. Prepositions, adverbs, nouns denoting spatial relations become the subject of special attention, comprehend, acquire generalized meaning in the learning process and, finally, contribute to the improvement of spatial orientation.

Children learn the vocabulary of temporary designations: morning, day, evening, night, yesterday, 
today, tomorrow, quickly, slowly, the names of the days of the week, months, seasons. Mastering the meaning of these words helps to comprehend "fluidity", duration, frequency of time, develops a "sense of time." With the help of words, not only are they reflected, but quantitative, spatial and temporal representations are more deeply realized and generalized. Speech enrichment also takes place due to the mastery of certain special terms (names of arithmetic operations, generally accepted units of measurement, geometric figures, etc.). Their volume is extremely insignificant, since the main content of children's speech is a "purely" household dictionary. In the formation of mathematical representations, speech development does not occur in isolation, but in conjunction with sensory and thought processes.

5. The formation of the initial forms of educational activity. An important role is played by pre-mathematical preparation for the formation of the initial forms of educational activity. Children develop the skills to listen and hear, act in accordance with the instructions of the teacher, understand and solve educational tasks in certain ways, use the didactic material for the purpose, express verbally the methods and results of their own actions and the actions of their comrades, monitor and evaluate them, to draw conclusions and generalizations, to prove their correctness and other skills and abilities of educational activity. The child masters mathematical representations mainly in the classroom, being in a peer team, thereby expanding the scope and experience of collective relationships between children. In the process of forming mathematical representations, preschoolers develop organization, discipline, the arbitrariness of mental processes and behavior, there is activity and interest in solving problems.

The noted tasks of pre-mathematical preparation of preschoolers take place in each group of kindergarten, but are specified taking into account age and individual characteristics. For its correct formulation and implementation, it is necessary for the teacher to know the development program of elementary mathematical representations not only of the group with which he works; the use of means, methods, forms and ways of organizing work that are adequate to the tasks and level of development of children; systematic work on the implementation of tasks both in the classroom for the formation of mathematical representations, and in everyday life.

Tasks are not solved in isolation, but in a complex, in close connection with each other. Being mainly aimed at the mathematical development of children, they are combined with the fulfillment of the tasks of moral, labor, physical and aesthetic education, i.e., the comprehensive development of the personality of preschool children. An integrated approach to their implementation is the most effective way of teaching young children. Tasks determine the content of pre-mathematical training in kindergarten.

The radical renewal of the content of upbringing and education in preschool organizations is due to the new educational policy of the Republic of Uzbekistan, aimed at reviving the cultural, creating role of education in society. The modern system of preschool education orientes society towards a new humanistic approach to the child as a developing personality, in need of understanding and respect for its interests and rights. Preschool childhood is an important stage in the formation of the moral character of a child. It is during these years that value orientations, the first moral ideas, feelings, habits, and relationships that determine the further development of the child's personality are formed [7].

Preschool education remains the world model of the first stage of continuous upbringing and early socialization of children, for the continuity of the educational process connects preschool, school and subsequent years of a child's life. Since the beginning of 2017, an active process of reforming the content of preschool education and training has been ongoing in the republic, including the specification of social and pedagogical functions based on the historical and cultural traditions of the Uzbek people and other ethnic groups living in the country [6] .

Analyzing the current state of preschool education in our country, it is advisable to consider not only positive, but also negative trends:

ever-increasing demands on the upbringing, training and development of children in the new sociocultural situation;

insufficient pre-school education for rural children;

decrease in the potential of the system of preschool education management, teacher training, as well as preschool teacher science;

insufficient involvement of adults and parents in the educational process;

lack of equal starting opportunities for preschool education of children with special educational needs;

insufficient equipment of the material and technical base in the organizations of preschool education and training;

Citation: Dzhumaev Mamanazar Irgashevich* and Urinbaeva .Use of Computer Software in Education of Students in Primary School and Preschool Op Acc J Bio Sci \& Res 7(1)-2021. 
lack of preschool industry.

The above trends indicate the need for the development of the Concept of preschool education and training.

The purpose of the concept is the development of scientific and methodological foundations of preschool education and training.

The concept of preschool education and training of the Republic of Uzbekistan includes the following sections:

a. The relevance of preschool education and training.

b. Methodological principles.

c. Goals and objectives of the content of preschool education and training.

d. The main directions of the content of preschool education and training.

e. Mechanisms for the implementation of the Concept of preschool education and training.

f. Conditions for the implementation of the Concept of preschool education and training.

g. Expected results of the content of preschool education and training.

\section{h. Conclusion}

The Relevance Of Updating The Content Of Preschool Education And Training

At the present stage, the system of preschool education in the Republic of Uzbekistan is being modernized in a number of areas:

determination of the conceptual foundations of the activities of the preschool education system;

introduction of the new State standard for preschool education and training;

the creation of scientific and programmatic support for preschool education;

updating goals, objectives, priorities, content, forms and methods, key results of preschool education.

The transition of the Uzbek school to the model of $10+$ 1-year education, the introduction of a competency- based approach to assessing educational outcomes at all levels of education necessitated updating the content of preschool education and training of the Republic of Uzbekistan. Innovative changes in the content of preschool education are caused by the following factors:

i. In accordance with changes in the surrounding world and the achievements of basic sciences, educational material is updated: new sections, topics, concepts necessary for life in modern society, of general cultural significance, are excluded and outdated sections and topics are excluded;

ii. In modern conditions, the education system is the main social environment for the younger generation. The main condition for the functioning of this environment is health saving. Health and a healthy lifestyle are recognized as the most important key results of quality education, and therefore, the valueological component should be included in the content of preschool education ;

iii. The influence on the content of preschool education of new means and technologies of activity has intensified, which has created a new learning environment for children from an early age. Modern information technologies constructively affect the content of preschool educational programs.

iv. The activity component of the content of preschool education is being developed : the mandatory minimum of the content of preschool education includes specially selected methods of activity, techniques and technologies, key competencies and other procedural elements that a preschooler needs to master.

Along with updating the content of preschool education, this Concept implies the further technologization of the pedagogical process, which will contribute to a different approach in preschool education, fundamentally changing the content of interaction between an adult (parent, teacher, educator) and a child. Today, technologies of personalityoriented, differentiated, projected learning, health-saving technologies, interactive, socio-game techniques and many others are being tested and effectively implemented in the process of educating and educating preschool children.

The use of new information and educational technologies by teachers leads to the creation of a specially organized development environment aimed at children acquiring certain knowledge, skills, competencies, in which the goals, content, methods and organizational forms of learning will be mobile and accessible for change within the framework of the preschool organization.

The innovative processes provided for in the content of preschool education are primarily aimed at creating a comfortable educational environment that allows children 
to be healthy, acquire psychological, personal confidence and successfully socialize in the world around them.

\section{Methodological Foundations and Principles}

The methodological basis of the study was:

i. regulatory legal provisions on the upbringing, training and development of preschool children;

ii. philosophical theories in the field of education;

iii. psychological concepts;

iv. pedagogical theories;

v. the work of foreign researchers;

vi. general scientific research methods;

vii. methodological approaches.

A systematic approach means the continuity of preschool education and training programs and the State compulsory standard of preschool education, which implies the interconnection of the components of the educational process. The personal- activity approach involves helping the child to become aware of himself as a person and contributes to self-affirmation in the process of including him in a variety of activities. A personalityoriented approach is based on the construction of a special kind of educational process (with specific goals, content, forms, methods and technologies), which is focused on the development and self-development of the child's personal properties. A competency-based approach allows you to identify the problem, make a responsible decision, determine the conditions necessary for its implementation, plan and organize the process of its achievement, evaluate and self-evaluate your activities and its results.

The concept of preschool education and training is based on the following principles:

1. The principle of development guides, stimulates and supports the emotional, spiritual, moral, intellectual development and self-development of the child, creates the conditions for the manifestation of independence, initiative, creative abilities of a preschooler in various activities.

2. The principle of nature-friendly education and training is based on a scientific understanding of the relationship between natural and sociocultural processes and the need for education and training of preschool children, taking into account their nature-like qualities, individual and age characteristics.

3. The principle of the humanistic orientation of upbringing and training presupposes the development of creative abilities, the strengthening of faith in oneself and the possibility of achieving an "ideal self" and establishes truly equal and partner relations with adults.

4. The principle of culture of conformity states that education and training should be based on universal values, be built in accordance with the traditions and norms of national culture and regional characteristics.

5. The principle of variability provides a wide variety of educational programs implemented in preschool organizations, aims to determine the individual trajectory of the development of the child's personality.

6. The principle of the value and uniqueness of a person is to recognize the intrinsic value of the person and the unique individuality of each child ("All children are loved - all children are equal!").

\section{Goal and Tasks}

updating the content of preschool education and training

Purpose: the establishment and development of pre-school education and training - the first stage of the continuing education system of the Republic of Uzbekistan on a competency basis;

\section{Tasks:}

i. Ensuring compliance of the content of preschool education and training with the expectations and educational needs of the state and society;

ii. ensuring the continuity of the content of preschool education and training and the content of education;

iii. updating the content of preschool education and training on a competency basis;

iv. inclusion of a valeological component in the content of preschool education (enrichment of the content of the educational field "Health" in order to ensure the protection of the life and health of the child, full physical development, the formation of values of a healthy lifestyle from an early age;

v. inclusion in the content of preschool education and training of a variable component (individual educational programs and trajectories within the educational areas of "Health", "Communication", "Cognition", "Creativity", "Socium") in accordance with nature-friendly qualities of a child's personality and for ensuring individual development of a child of preschool age; 
vi. inclusion in the content of preschool education and training of the most important moral norms and provisions with the aim of forming a preschool child's valuable moral orientations, fostering a humane attitude to himself, to his family, to people, to the world around him;

vii. inclusion in the content of pre-school education and training of the humanitarian component (primarily enrichment of the content of the educational areas "Health", "Communication", "Cognition", "Creativity" with knowledge from the humanities), a multilingual component (state, Russian, foreign languages) in order to form the spiritual potential of the individual, the multilingual capabilities of the child of preschool age and ensure the effective socialization of the child in modern society;

viii. ensuring the holistic content of educational programs for pre-school education and training with the aim of creating a full-fledged developing space and providing comprehensive support for the individual development of a young child.

\section{Key Areas for Updating Content}

\section{pre-school education and training}

\section{Health and physical development of the child:}

i. To educate children the interest and desire to lead a healthy lifestyle;

ii. instill cultural and hygienic skills;

iii. educate children in a careful attitude to their health;

iv. improve motor activity of children,

v. develop interest in various sports;

vi. develop physical qualities (will, determination, endurance, courage).

\section{Communicative-Linguistic Development}

i. improve and enrich the speech of a preschooler;

ii. To raise interest in the state language and the need to learn it;

iii. use a variety of means of verbal communication in their native and other languages to build relationships and interaction;

iv. To raise interest in the word of art and respect for the book;

v. to form a culture of communication and ensure the interaction of preschoolers in the process of joint activities with peers and adults;

vi. to educate the multilingual personality of a preschooler who has mastered his native language, is ready to interact in the state and other languages, able to communicate with the outside world through verbal and non-verbal means.

\section{Cognitive Development}

i. to form in children a system of elementary knowledge about objects and phenomena of life as a condition for mental growth;

ii. develop skills and cognitive activities;

iii. form a system of basic mental actions (analysis, synthesis, comparison, generalization, classification, etc.);

iv. to develop in children of preschool age curiosity, initiative, independence in obtaining new impressions, search and testing of alternative different methods of action, finding answers to questions and solving problem situations;

v. to expand the general ideas of the child about the world, himself, other people.

\section{Social and Personal Development}

i. To cultivate a humanistic attitude to the surrounding social and natural world;

ii. cultivate respect for the symbols of the Republic of Uzbekistan (coat of arms, flag, anthem);

iii. to form moral qualities in preschoolers (feelings of national pride, humane attitude to people, moral behavior);

iv. To cultivate love for their people, their spiritual culture and the culture of the nationalities living in the Republic of Uzbekistan ;

v. Tocultivate tolerant behavior through harmonization of relations: child $\leftrightarrow$ child, child $\leftrightarrow$ adult, kindergarten $\leftrightarrow$ family, family $\leftrightarrow$ child $\leftrightarrow$ kindergarten $\leftrightarrow$ society;

vi. Form a responsible attitude to the family, their people and the Republic of Uzbekistan ;

vii. familiarize children with the history and culture of the Uzbek people and the peoples living in the country;

viii. to form a positive attitude to work, a responsible and creative attitude to different types of labor;

ix. To cultivate a caring attitude to adult work, to 
people of any profession, awareness of the importance of their work for others.

\section{Creative Education}

i. to form an artistic and aesthetic attitude of children to the environment;

ii. acquaint children with different types, genres of art, Uzbek folk art;

iii. to form artistic and aesthetic representations, concepts and judgments ;

iv. to introduce children to active artistic and aesthetic activities;

v. to develop the artistic and aesthetic perception of children through the knowledge of the customs, traditions and life of the Uzbek people and other ethnic groups inhabiting the republic ;

vi. to develop in preschoolers with the help of the game the need to transform the surrounding reality, the ability to create new things;

vii. form ideas about the world, develop taste and moral feelings with the help of children's toys;

viii. develop the creative abilities of the child in various types of children's games.

\section{Work with Parents}

i. organize interaction with the family in a triad of subjective relations "child + parent + teacher";

ii. assist parents in mastering the methods and technologies of raising children of preschool age;

iii. help parents acquire pedagogical knowledge in order to form the qualities of an educator;

iv. establish an open and trusting relationship with parents based on:

v. honest and open communication;

vi. mutually agreed educational goals;

vii. open bilateral cooperation.

\section{Mechanisms for Implementing The Development}

Concept

\section{pre-school education and training}

Training of highly qualified teaching staff.

Material and technical equipment of preschool organizations.

Software and methodological support.

The introduction of new educational and information technologies.

Material and moral stimulation of participants in the pedagogical process.

\section{In Words the Realization of the Concept of Preschool Education}

Organizational and managerial support consists in creating comfortable conditions for the upbringing and education of preschool children; in the study, generalization and dissemination of pedagogical experience of the best educators. Scientific and methodological support is carried out by improving the qualifications of teachers in the system of preschool education. Definition of the conceptual foundations of preschool education. Development and introduction of a scientific base for modernization in the processes in the system of preschool education. Development and implementation of variable programs, educational and information technologies.

Psychological and pedagogical support consists in partnership and cooperation of teachers - educators with the teaching staff of pedagogical universities and colleges, the community of parents and public organizations in preschool education of children.

\section{Expected Content Updates}

pre-school education and training

Formation in the Republic of Uzbekistan of an integrated system of preschool competency-based education - the first stage of the national education system, to the greatest extent:

i. taking into account the educational needs of the state, society, various categories of the population, the personality of the child;

ii. providing high-quality educational and additional services;

iii. effectively adapting to the changing conditions of the modern world.

Quite honestly, many elementary teachers are much more comfortable teaching literacy than they arContainers in elementary classroom representing place value

Approaches to teaching place value differ depending upon grade level. In the primary grades, place value is taught using many techniques; one of the most-effecive is using 
base-10 manipulatives, which come in different shapes for different place values. After the focus of the early grades, in general, students possess an understanding of place value by the time they arrive in the intermediate grades. As a result, we are no longer focusing on identifying 1's, 10's, or 100's places in a number; instead, we focus on applying place-value understanding for everyday applications and solving problems. Simple games for repetition All students need to understand how to apply place value to verbally say a large number. This is tough for students and takes a lot of repetition and practice. But repetition can be fun! Here's how I handle it in my classroom.

This "game" is simple and can be fit in between other elementary math activities. I write a large number on the board, something in the millions or even higher if I feel they need more of a challenge.

Then in the morning, as they're putting their personal whiteboards away after number strings, they must file by me and read the number correctly. If they don't read it correctly, they go to the end of the line and come back through.

The children love it! It's a fun challenge, but most importantly they get a little bit of that one-on-one time with their teacher, which is always a huge motivatore teaching math. Literacy is like a sprawling vine that covers all kinds of different skills. Therefore, it can be taught through multiple methods and generally arrive at the same goal. Math, on the hand, is very linear/sequential. It must be taught in a logical and organized, step-by-step approach. A lot of elementary teachers are not comfortable with that and tend to move on before the children have an extremely solid understanding of foundational concepts. Of course, readers of my website will understand that I believe our future mathematicians will suffer from this approach... as will your end-of-year test scores! The articles in this section will help you create great mathematicians who will be well-positioned for success in middle and high school.

\section{Comparing Numbers}

Place value in intermediate grades really comes into play when we are comparing two numbers. For example, when comparing the number 400,000 with the number 400 , students need to be able to express how much bigger the " 4 " is in the first number than it is in the second number (it's 1,000 times bigger).I often mix in other numbers just to confuse them, such as having to compare 435,672 with 419 . Of course, the answer is the same.

When teaching place value, we are trying to instill an awareness of size versus an awareness of digits. The digits are simply mathematical symbols that must be placed within the context of their position.

\section{Conclusion}

The concept of development of preschool education and training is the most important component of the modernization of the national system of preschool education, due to a number of factors:

i. updating the role and purpose of preschool education in the system of continuing education;

ii. updating goals, objectives, principles, approaches, priorities in the system of preschool education;

iii. A new vision of the key results of preschool education;

iv. transition to a competency-based model of preschool education.

This Concept outlined the strategic guidelines for the development of preschool education and training, following which will contribute to:

1. Improving the resource support of the process of education and training of preschool children (financial, material, technical, personnel, software, scientific and methodological, technological);

2. Modeling of a high-quality, developing, healthsaving, sociocultural environment in preschool organizations;

3. Technologization of the educational process in preschool organizations;

\section{achieving the quality of preschool education in the Republic of Uzbekistan.}

Foreign language lesson has its own specificity, unlike other school subjects; the main objective of a foreign language lesson is the formation of intercultural communicative competence of learners. At the present moment the global aim of teaching foreign language is involvement to other culture and participation in dialogue of cultures. This aim is gained by the way of formation the ability to intercultural communication. The process of teaching foreign language is organized on the basis of communicative character tasks; teaching foreign language communication using all necessary for this work means is a distinctive feature of a foreign language lesson. 


\section{References}

1. Mirziyoyev Sh M (2017) Ensuring the rule of law and human interests is a guarantee of the country's development and the well-being of the people. Tashkent, Uzbekistan 48.

2. Mirziyoyev Sh M (2017) Critical analysis, tough distsipline and personal responsibility should become the everyday norm in the activities of each leader. Tashkent, Uzbekistan 104.

3. Strategy of action in five priority areas of the development of the Republic of Uzbekistan in 2017-2021. to the Decree of the President of the Republic of Uzbekistan dated 4947.

4. Driga VI (2012) Development of the professional career of a modern teacher in conditions of creative education / V.I. Driga // Standards and monitoring in education 4: 48-51

5. Loktionova TE, Sergeev MG (2018) A comfortable educational environment in an educational organization: a modern approach to design. Vocational education and society 3(27): 43-106.

6. Davydov VV (1992) The psychological theory of learning activity and IU todov primary education, based on meaningful generalizations Research Institute. Tomsk 112.

7. Peterson LG (1984) Modeling as a means of formation before the representations of the concept of function in 4-6 classes of high school. Dis . for a job . student step. Cand. ped . sciences 201.

8. Jinho Kim (2013) Mathematics Education in Korea: Curricular and Teaching and Learning Practices. World Scientific.

9. Mathematics syllabuses (2015) Cirriculum planning and development division, Singapore Ministry of education.

10. Character and Citizenship Syllabuses (2016) Framework for 21st Century competencies and Student outcomes. Student development cirriculum division, Singapore Ministry of education.

11. The National School Cirriculum, Cirriculum and Assesment Guide. CDC-HKEAA Committee. Hong Kong.

12. Ginsburg A, Leinwand S, Decker K (2009) Informing Grades 1-6 Standards Development: What Can Be Learned from
High-Performing Hong Kong, Korea, and Singapore?" American Institutes for Research.

13. California Common Core State Standards (2013) Mathematics. Electronic Edition. Adopted by the California State Board of Education August 2010 and modified.

14. Zhumaev ME (2016) Methods of teaching mathematics for elementary grades: a Textbook. Turon Ikbol. Tashkent 426.

15. Davydov VV (1992) The psychological theory of educational activities and methods of elementary education, based on a meaningful generalization. - Tomsk 112.

16. Peterson LG (1984) Modeling as a means of forming ideas about the concept of function in grades 4-6 of high school. Dis. for a job. student step. Cand. ped sciences 20.

17. Ivankova N, Zhumagulova N, Povstjan L, Alkhatova T, Sakenov J (2016) Studying teachers 'and parents' attitudes towards educationally underperforming adolescents - Students of general education schools. New Educational Review 44(2): 62-71.

18. Makhashova P, Meirmanov A, Zhunusbekov Z, Ermuratova A, Sakenov J (2016) On the development of professional competence in students of creative pedagogical specialties. International Journal of Environmental and Science Education11(11): 46604668.

19. Omarov YB, Toktarbayev DG, Rybin IV, Baitlessova N, Sakenov J (2016) Methods of forming professional competence of students as future teachers. International Journal of Environmental and Science Education 11(14): 6651-6662.

20. Nurgazina AB, Rakhimzhanov KH, Akosheva MK, Baratova MN, Sakenov J (2016) About the poetic text and a concept as ways of representation of author's subjectivity and modality. International Journal of Environmental and Science Education 11(18): 11757-11770.

21. Zhumaev MI Formation of the didactic business skillsof the teacher in elementary school Department of primary education,Tashkent State Pedagogical University named after Nizami, Tashkent city, Republic of Uzbekistans

*Corresponding author: Dzhumaev Mamanazar Irgashevich, Email: mamanazaruz@bk.ru

Next Submission with BGSR follows:

- Rapid Peer Review

- Reprints for Original Copy

- E-Prints Availability

- Below URL for auxiliary Submission Link: https://biogenericpublishers.com/submit-manuscript/ 